SISSA $170 / 92 /$ EP

\title{
NEUTRINOS WITH MIXING IN TWISTING MAGNETIC FIELDS
}

\author{
In memoriam of Ya.A. Smorodinsky \\ E.Kh. Akhmedov 田, S.T. Petcov 自色 \\ Scuola Internazionale Superiore di Studi Avanzati \\ Strada Costiera 11, I-34014 Trieste, Italy \\ and \\ A.Yu. Smirnov \\ Institute for Nuclear Research, Russian Academy of Sciences, Moscow, Russia, and \\ International Centre for Theoretical Physics, I-34100 Trieste, Italy
}

\begin{abstract}
Transitions in a system of neutrinos with vacuum mixing and magnetic moments, propagating in matter and transverse magnetic field, are considered. It is shown that in the realistic case of magnetic field direction varying along the neutrino path qualitatively new phenomena become possible: permutation of neutrino conversion resonances, appearance of resonances in the neutrino-antineutrino $\left(\nu_{l L} \leftrightarrow \bar{\nu}_{l R}\right)$ transition channels, neutrino-antineutrino resonant conversion, large amplitude $\nu_{l L} \leftrightarrow \bar{\nu}_{l R}$ oscillations, merging of different resonances (triple resonances). Possible phenomenological implications of these effects are briefly discussed.
\end{abstract}

*On leave from Kurchatov Institute of Atomic Energy, Moscow 123182, Russia

†E-mail: akhmedov@tsmi19.sissa.it, akhm@jbivn.kiae.su

${ }^{\ddagger}$ Istituto Nazionale di Fisica Nucleare, Sezione di Trieste, Trieste, Italy

$\S$ Permanent address: Institute of Nuclear Research and Nuclear Energy, Bulgarian Academy of Sciences, BG-1784 Sofia, Bulgaria 


\section{Introduction}

Neutrinos with magnetic or transition magnetic moments $(\mu)$ propagating in transverse magnetic fields $\left(\mathbf{B}_{\perp}\right)$ will undergo spin precession [1, 2, 3] or spin-flavour precession [4, 3], 5], 6], respectively. As is well known, both types of precession can have important astrophysical implications. In the presence of matter, the neutrino spin-flavour precession can be resonantly enhanced [5, 6]. The resonant spin-flavour precession (RSFP) is analogous to the MSW effect in neutrino oscillations [7, 8]. If neutrino mixing exists, an interplay between the RSFP and the MSW effect is possible. In particular, the matter-enhanced neutrino oscillations can assist the RSFP in giving rise to time variations of the solar neutrino flux by improving the adiabaticity of the latter [9].

In most of the previous studies of the spin and spin-flavour precessions of neutrinos in transverse magnetic fields it was assumed that the direction of the field is fixed. However, in the physically interesting cases of three-dimensional field configurations (e.g., in the Sun, supernovae, etc.), neutrinos traversing the magnetic field will feel, in general, a field whose direction is changing along the neutrino trajectory [10, 11, 12, 13, 11]. The presence of such twisting magnetic fields can lead to new interesting phenomena. In particular, the neutrino spin precession can be resonantly enhanced, the energy dependence of the probability of the resonant spin-flavour precession can be significantly changed, the lower limit on the value of $\mu B_{\perp}$ required to account for the solar neutrino problem can be slightly relaxed and, in addition, new realizations of RSFP become possible [12, 13].

The RSFP of neutrinos in twisting magnetic fields was considered so far under the simplifying assumption of absence of neutrino mixing in vacuum. In the present paper we study the general case: we assume the existence not only of flavour-off-diagonal neutrino magnetic moments, but also of flavour neutrino mixing in vacuum. This is a natural possibility if no additive lepton number is conserved. Under the above condition both resonant oscillations and RSFP of neutrinos are allowed. We show that if they take place in twisting magnetic fields, qualitatively new phenomena become possible: resonant enhancement of the helicity- 
flipping $\Delta L_{l}=2$ neutrino-antineutrino conversions $\nu_{l L} \leftrightarrow \bar{\nu}_{l R}(l=e, \mu, \tau)$, and merging of three different transition resonances. Moreover, for a definite sign of the angular velocity of the magnetic field rotation, the order of the RSFP and MSW resonances in matter with monotonically decreasing density can change. All these effects can have an important impact on solar and supernova neutrinos.

\section{Neutrino Evolution Equations}

Consider a system of two flavour neutrinos and their antiparticles, say $\nu_{e L}, \bar{\nu}_{e R}, \nu_{\mu L}$, and $\bar{\nu}_{\mu R}$. We shall assume that $\nu_{e}-\nu_{\mu}$ mixing is generated by a Majorana neutrino mass term, and that there exists a $\nu_{e L}-\bar{\nu}_{\mu R}$ transition magnetic moment $\mu$. The equations for the flavour neutrino wave functions, describing the propagation of neutrinos in matter and transverse magnetic field in the case of interest, can be written in the form:

$i \frac{d}{d t}\left(\begin{array}{c}\nu_{e L} \\ \bar{\nu}_{e R} \\ \nu_{\mu L} \\ \bar{\nu}_{\mu R}\end{array}\right)=\left(\begin{array}{cccc}N(t)-c_{2} \delta & 0 & s_{2} \delta & \mu B_{\perp}(t) e^{i \phi(t)} \\ 0 & -N(t)-c_{2} \delta & -\mu B_{\perp}(t) e^{-i \phi(t)} & s_{2} \delta \\ s_{2} \delta & -\mu B_{\perp}(t) e^{i \phi(t)} & -r(t) N(t)+c_{2} \delta & 0 \\ \mu B_{\perp}(t) e^{-i \phi(t)} & s_{2} \delta & 0 & r(t) N(t)+c_{2} \delta\end{array}\right)\left(\begin{array}{c}\nu_{e L} \\ \bar{\nu}_{e R} \\ \nu_{\mu L} \\ \bar{\nu}_{\mu R}\end{array}\right)$

Here the angle $\phi(t)$ defines the direction of the magnetic field $\mathbf{B}_{\perp}(t)$ in the plane orthogonal to the neutrino momentum, $B_{\perp}(t)=\left|\mathbf{B}_{\perp}(t)\right|$,

$$
\begin{gathered}
N \equiv \sqrt{2} G_{F}\left(n_{e}-n_{n} / 2\right), \quad r \equiv \frac{n_{n}}{2 n_{e}-n_{n}}, \quad \delta \equiv \Delta m^{2} / 4 E \\
s_{2} \equiv \sin 2 \theta_{0}, \quad c_{2} \equiv \cos 2 \theta_{0}
\end{gathered}
$$

where $G_{F}$ is the Fermi constant, $n_{e}$ and $n_{n}$ are the electron and neutron number densities, $E$ is the neutrino energy, $\Delta m^{2}=m_{2}^{2}-m_{1}^{2}, m_{1}$ and $m_{2}$ being the masses of two Majorana neutrinos and $\theta_{0}$ being the neutrino mixing angle in vacuum. The zeros in the effective Hamiltonian in eq. (1) are related to the fact that diagonal magnetic moments of Majorana neutrinos are precluded by $C P T$ invariance. 
It is instructive to consider the evolution of the neutrino system in the reference frame which rotates with the same angular velocity as the transverse magnetic field [12]. The corresponding transformation of the neutrino wave functions reads: $\nu=S \nu^{\prime}$, where $\nu$ is the column vector of state of the neutrino system in eq. (1), and $S=\operatorname{diag}\left(e^{i \frac{\phi}{2}}, e^{-i \frac{\phi}{2}}, e^{i \frac{\phi}{2}}, e^{-i \frac{\phi}{2}}\right)$. Since $S$ is a diagonal matrix, the above transformation does not change the probabilities of the neutrino transitions and therefore we will keep the same notations for the neutrino wave functions in the new frame. In the rotating frame the phase factors disappear from the nondiagonal terms (see eq. (1)) and the evolution equations take the form:

$i \frac{d}{d t}\left(\begin{array}{c}\nu_{e L} \\ \bar{\nu}_{e R} \\ \nu_{\mu L} \\ \bar{\nu}_{\mu R}\end{array}\right)=\left(\begin{array}{cccc}N-c_{2} \delta+\frac{\dot{\phi}}{2} & 0 & s_{2} \delta & \mu B_{\perp} \\ 0 & -N-c_{2} \delta-\frac{\dot{\phi}}{2} & -\mu B_{\perp} & s_{2} \delta \\ s_{2} \delta & -\mu B_{\perp} & -r N+c_{2} \delta+\frac{\dot{\phi}}{2} & 0 \\ \mu B_{\perp} & s_{2} \delta & 0 & r N+c_{2} \delta-\frac{\dot{\phi}}{2}\end{array}\right)\left(\begin{array}{c}\nu_{e L} \\ \bar{\nu}_{e R} \\ \nu_{\mu L} \\ \bar{\nu}_{\mu R}\end{array}\right)$

where $\dot{\phi} \equiv d \phi / d t$.

The $4 \times 4$ matrix on the right-hand side of the Schroedinger-like equation (3) defines the time-dependent effective Hamiltonian of the system, $H$. Note that the effect of rotation of the magnetic field amounts to the appearance of additional terms of the same absolute value in the diagonal elements of $H$. These terms have the same signs for the neutrinos possessing the same helicity and opposite signs for the neutrinos of opposite helicities. The indicated features have a simple physical interpretation [13]. According to classical mechanics, a system with angular momentum $\mathbf{s}$ acquires an additional energy $\Delta E=-(\mathbf{s} \Omega)$ upon a transformation to a reference frame rotating with an angular velocity $\Omega$. In our case $\Omega=\dot{\phi}$, $s=1 / 2$, and the projections of $\mathbf{s}$ on $\Omega$ are equal to $-1 / 2$ for the neutrinos, and to $+1 / 2$ for the antineutrinos.

Let us consider the resonant properties of the neutrino system under study. The diagonal elements of the Hamiltonian in eq. (3) define the energies of the "flavour levels", i.e. of $\nu_{e L}, \bar{\nu}_{e R}, \nu_{\mu L}$, and $\bar{\nu}_{\mu R}$, in the absence of vacuum mixing and magnetic field; we shall denote them respectively as $H_{e}, H_{\bar{e}}, H_{\mu}$, and $H_{\bar{\mu}}$. Resonances take place at densities at which 
the splittings between the eigenvalues of the effective Hamiltonian $H$ with $s_{2} \delta \neq 0$ and $\mu B_{\perp} \neq 0$ are minimal. The resonance densities can be approximately found by equating pairs of diagonal elements of $H: H_{\alpha}\left(n_{e}, n_{n}\right)=H_{\beta}\left(n_{e}, n_{n}\right), \alpha \neq \beta, \alpha, \beta=e, \bar{e}, \mu, \bar{\mu}$ (i.e. they correspond approximately to the values of $N$ at which the flavour levels cross).

With four neutrino states there are altogether six resonance conditions, and in what follows we will consider the nature and the properties of the corresponding resonances.

Two conditions, $H_{e}=H_{\mu}$ and $H_{\bar{e}}=H_{\bar{\mu}}$, can be written as:

$$
\begin{aligned}
\sqrt{2} G_{F} n_{e} & =\frac{\Delta m^{2}}{2 E} \cos 2 \theta_{0} \quad\left(\nu_{e L} \leftrightarrow \nu_{\mu L}\right), \\
\sqrt{2} G_{F} n_{e} & =-\frac{\Delta m^{2}}{2 E} \cos 2 \theta_{0} \quad\left(\bar{\nu}_{e R} \leftrightarrow \bar{\nu}_{\mu R}\right) .
\end{aligned}
$$

They concern the transitions (given in parentheses) between neutrinos having the same helicity but different flavour, and thus correspond to the MSW resonances. The condition for the neutrinos (antineutrinos) can be satisfied for $\Delta m^{2} \cos 2 \theta_{0}>0\left(\Delta m^{2} \cos 2 \theta_{0}<0\right)$. Eqs. (4) and (5) do not depend on $\dot{\phi}$ since the flavour neutrino states involved have the same helicity and the magnetic field rotation influences both states equally. Magnetic fields (twisting or nontwisting) do not modify the MSW resonances.

The next two conditions, $H_{e}=H_{\bar{\mu}}$ and $H_{\bar{e}}=H_{\mu}$, have the form:

$$
\begin{aligned}
\sqrt{2} G_{F}\left(n_{e}-n_{n}\right) & =\frac{\Delta m^{2}}{2 E} \cos 2 \theta_{0}-\dot{\phi} \quad\left(\nu_{e L} \leftrightarrow \bar{\nu}_{\mu R}\right), \\
\sqrt{2} G_{F}\left(n_{e}-n_{n}\right) & =-\frac{\Delta m^{2}}{2 E} \cos 2 \theta_{0}-\dot{\phi} \quad\left(\bar{\nu}_{e R} \leftrightarrow \nu_{\mu L}\right) .
\end{aligned}
$$

The neutrinos involved in the relevant transitions have different flavours, as well as opposite helicities. Thus, eqs. (6) and (7) define the resonance conditions for the spin-flavour precession: eq. (6) refers to the channel $\nu_{e L} \leftrightarrow \bar{\nu}_{\mu R}$, while eq. (7) refers to the $C P$-conjugated one. Since the neutrinos involved have different helicities, the magnetic field rotation modifies the resonance conditions: both eq. (6) and eq. (7) depend on $\dot{\phi}$. In a medium with $\left(n_{e}-n_{n}\right)$ of a definite sign (like the matter of the Sun) and $\dot{\phi}=0$ only one of these two conditions can be satisfied depending on the sign of $\Delta m^{2} \cos 2 \theta_{0}$. In contrast, in the case of twisting 
magnetic field both conditions can be obeyed simultaneously. This can happen for $\dot{\phi}<0$ $(\dot{\phi}>0)$ when $n_{e}>n_{n}\left(n_{e}<n_{n}\right)$ provided $|\dot{\phi}|>\left|\left(\Delta m^{2} / 2 E\right) \cos 2 \theta_{0}\right|$. Obviously, the two conditions will be fulfilled at different densities.

Finally, equating the elements $H_{e}=H_{\bar{e}}$ and $H_{\mu}=H_{\bar{\mu}}$ one finds:

$$
\begin{aligned}
\sqrt{2} G_{F}\left(n_{e}-n_{n} / 2\right) & =-\dot{\phi} / 2 \quad\left(\nu_{e} \leftrightarrow \bar{\nu}_{e}\right), \\
\sqrt{2} G_{F}\left(-n_{n} / 2\right) & =-\dot{\phi} / 2 \quad\left(\nu_{\mu} \leftrightarrow \bar{\nu}_{\mu}\right) .
\end{aligned}
$$

Equations (8) and (9) represent the resonant conditions for the channels $\nu_{e L} \leftrightarrow \bar{\nu}_{e R}$ and $\nu_{\mu L} \leftrightarrow \bar{\nu}_{\mu R}$, respectively. In these transitions the helicity is flipped and the electron or the muon lepton number is changed by two units. Thus, we deal here with a qualitatively new phenomenon: neutrino-antineutrino helicity flipping $\Delta L_{l}=2$ resonance conversions.

In a medium with nonvanishing $\left(n_{e}-n_{n} / 2\right)$ and $n_{n}$ (like the Sun's matter) conditions (8) and (9) cannot hold in a nonrotating magnetic field. For fixed direction of the field rotation only one of these two conditions can be fulfilled: if, for example, $n_{e}>n_{n} / 2$, for $\dot{\phi}<0$ the resonance takes place in the electron neutrino channel, $\nu_{e L} \leftrightarrow \bar{\nu}_{e R}$, whereas for $\dot{\phi}>0$ it occurs in the muon neutrino channel, $\nu_{\mu L} \leftrightarrow \bar{\nu}_{\mu R}$.

It should be emphasized that the neutrino-antineutrino transitions are forbidden in the lowest order of the interactions described by the effective Hamiltonian in eq. (3): the matrix elements of $H$ which mix neutrino and antineutrino states of the same flavour are zero. As we have mentioned earlier, this follows from the fact that Majorana neutrinos cannot have nonzero diagonal magnetic moment. In Section 4 we will show that the mixing between neutrinos and antineutrinos is induced in the second order of the perturbation theory in the interactions described by $H$. 


\section{Merging and Permutation of Resonances}

The energies of the flavour neutrino levels, $H_{\alpha}$, have different $\dot{\phi}$ dependence. Consequently, the resonance densities associated with the various neutrino transitions are different functions of $\dot{\phi}$. Therefore the magnetic field rotation modifies the level crossing scheme. In particular, one can expect such effects as reduction (or increase) of the spatial distance between the resonances, or merging of resonances, or permutation of resonances, to take place.

In what follows we shall consider the dependence of the level crossing scheme on the

value of $\dot{\phi}$. We shall assume for simplicity that the chemical composition of the medium does not change appreciably along the neutrino path, so that $r(t) \cong$ const. In this case the level energies $H_{\alpha}$ depend on one density parameter only: $N(t)$. It can take both positive and negative values; in particular, $N(t)$ is always positive in the Sun, but can be negative in a strongly neutronized medium like the one existing in the central regions of collapsing stars.

It proves convenient to subtract the matrix $H_{e} \cdot I$, proportional to the unit matrix $I$, from the Hamiltonian $H$ in eq. (3). This is equivalent to a multiplication of all wave functions by the same phase factor $\exp \left(-i H_{e} t\right)$ which, obviously, does not change the neutrino transition probabilities. The diagonal elements of the Hamiltonian $\left(H-H_{e} \cdot I\right) \operatorname{read}$ :

$$
\left\{H_{e}, H_{\bar{e}}, H_{\mu}, H_{\bar{\mu}}\right\}=\left\{0,-2 N-\dot{\phi},-(1+r) N+2 c_{2} \delta,-(1-r) N+2 c_{2} \delta-\dot{\phi}\right\} .
$$

The resonance values of the density parameter $N$, corresponding to the different neutrino transitions, can be easily deduced from eq. (10). For flavour neutrino transitions one has:

$$
N_{f(\bar{f})}= \pm \frac{2 c_{2} \delta}{1+r}
$$

where the plus sign corresponds to the $\nu_{e L} \leftrightarrow \nu_{\mu L}$ channel, and the minus sign, to the $C P$ conjugated one $\bar{\nu}_{e R} \leftrightarrow \bar{\nu}_{\mu R}$. The resonance values of $N$ for the spin-flavour conversions are 
equal to

$$
N_{s f(\overline{s f})}=\frac{ \pm 2 c_{2} \delta-\dot{\phi}}{1-r}
$$

where the plus (minus) sign corresponds to the $\nu_{e L} \leftrightarrow \bar{\nu}_{\mu R}\left(\bar{\nu}_{e R} \leftrightarrow \nu_{\mu L}\right)$ transitions. Finally, the resonances in the neutrino-antineutrino transitions $\nu_{e L} \rightarrow \bar{\nu}_{e R}$ and $\nu_{\mu L} \rightarrow \bar{\nu}_{\mu R}$ take place respectively for

$$
\begin{gathered}
N_{e \bar{e}}=-\frac{\dot{\phi}}{2}, \\
N_{\mu \bar{\mu}}=\frac{\dot{\phi}}{2 r} .
\end{gathered}
$$

We shall assume for simplicity that $\dot{\phi}=$ const along the neutrino trajectory. Then the energy levels $H_{\alpha}=H_{\alpha}(N)$ are straight lines (see Figs. 1 and 2). After the phase transformation of the neutrino wave functions i) $H_{e} \equiv 0$ by definition, and ii) the muon neutrino energy level $H_{\mu}$ does not depend on $\dot{\phi}$. The antineutrino energy levels $H_{\bar{e}}$ and $H_{\bar{\mu}}$ have the same $\dot{\phi}$ dependence. Consequently, when $\dot{\phi}$ varies, $H_{e}$ and $H_{\mu}$ do not change, whereas $H_{\bar{e}}$ and $H_{\bar{\mu}}$ are shifted by the same amount. Finally, note that for $r<1$ (which is realized in the Sun) the strongest dependence on $N$ is exhibited by $H_{\bar{e}}$, the dependence of $H_{\mu}$ being somewhat weaker, while that of $H_{\bar{\mu}}$ is the weakest.

We shall assume that neutrinos are propagating in matter with density decreasing monotonically along the neutrino trajectory. Consider first the possibility $c_{2} \delta>0$. Let us recall the level crossing scheme in the case of nonrotating magnetic field. With $\dot{\phi}=0$ (Fig. 1) there are only two resonances for $N>0$ : the spin-flavour, $\nu_{e L} \leftrightarrow \bar{\nu}_{\mu R}$, and the flavour (i.e. MSW) one, $\nu_{e L} \leftrightarrow \nu_{\mu L}$. The spin-flavour resonance is located at higher density as compared to the density of the MSW resonance [6, 15]:

$$
\frac{N_{s f}}{N_{f}}=\frac{1+r}{1-r}>1
$$

Therefore if $\nu_{e L}$ is produced at a density $N_{0}>N_{s f}$ and the adiabaticity condition is fulfilled at the spin-flavour resonance, $\nu_{e L}$ will be converted into $\bar{\nu}_{\mu R}$ (provided the resonances do not overlap). If $N_{0}<N_{s f}$, or if the adiabaticity at the spin-flavour resonance is strongly 
violated, $\nu_{e L}$ will be transformed completely or partially into $\nu_{\mu L}$, depending on the degree of adiabaticity at the flavour resonance $\left(N=N_{f}\right)$.

Suppose now that $\dot{\phi} \neq 0$. The effects caused by $\dot{\phi}$ being nonzero depend on the direction of the field rotation and we shall discuss first the case $\dot{\phi}<0$. With $|\dot{\phi}|$ increasing, the $\bar{\nu}_{\mu R}$ and $\bar{\nu}_{e R}$ energy levels become higher and the following changes take place.

a) $0<|\dot{\phi}|<2 c_{2} \delta$ (Fig. 2a). A $\nu_{e L}-\bar{\nu}_{e R}$ level crossing appears for $N>0$, so that under certain conditions (see Section 4) $\nu_{e L}$ can undergo a resonant conversion into $\bar{\nu}_{e R}$.

b) $2 c_{2} \delta<|\dot{\phi}|<4 c_{2} \delta /(1+r)$ (Fig. 2b). For $N>0$ a resonance appears in the $\nu_{\mu L}-\bar{\nu}_{e R}$ channel. This means that two different types of spin-flavour conversion can take place in a medium with $N>0: \nu_{e L} \leftrightarrow \bar{\nu}_{\mu R}$, and $\nu_{\mu L} \leftrightarrow \bar{\nu}_{e R}$. Moreover, in such a medium $\nu_{e L}$ can be transformed into $\bar{\nu}_{e R}$ via two consecutive resonant conversions: first the MSW one $\nu_{e L} \rightarrow \nu_{\mu L}\left(\right.$ at $\left.N=N_{f}\right)$ and then the spin-flavour one $\nu_{\mu L} \rightarrow \bar{\nu}_{e R}\left(\right.$ at $\left.N=N_{\overline{s f}}\right)$. We shall call this process a two-step $\nu_{e L} \rightarrow \bar{\nu}_{e R}$ resonant conversion.

c) At $\dot{\phi}=\dot{\phi}_{1 m}$, where

$$
\dot{\phi}_{1 m} \equiv-\frac{4 c_{2} \delta}{1+r}
$$

(Fig. 2c), three resonances merge in one point: $N_{f}=N_{\overline{s f}}=N_{e \bar{e}}=2 c_{2} \delta /(1+r)$ (see eqs. (11)-(13)). In particular, the two resonances present in the two-step $\nu_{e L} \rightarrow \bar{\nu}_{e R}$ transition merge and a strong $\nu_{e L} \rightarrow \bar{\nu}_{e R}$ conversion can take place in one resonant region (see Section $5)$.

d) For $|\dot{\phi}|>\left|\dot{\phi}_{1 m}\right|$ (Fig. 2d) the flavour and the spin-flavour resonances permute and if $N_{0}<N_{s f}$, a $\nu_{e L}$ propagating from high to low densities will cross first the $\nu_{e L} \leftrightarrow \bar{\nu}_{e R}$ resonance and later the $\nu_{e L} \leftrightarrow \nu_{\mu L}$ one. If the adiabaticity condition is fulfilled, $\nu_{e L}$ will undergo efficient conversion into $\bar{\nu}_{e R}$ in the direct $\nu_{e L} \rightarrow \bar{\nu}_{e R}$ transition before reaching the MSW resonance (for which the adiabaticity parameter can be much bigger).

For large values of $|\dot{\phi}|$ the energy level $H_{\bar{\mu}}$ is far away from the energy domain where the $\nu_{e L}, \nu_{\mu L}$ and $\bar{\nu}_{\mu R}$ levels cross, so that in the corresponding density region the $\bar{\nu}_{\mu R}$ decouples 
from the dynamics of the other three neutrinos (see further, Section 5).

For positive values of $\dot{\phi}$ the antineutrino energy levels $H_{\bar{e}}$ and $H_{\bar{\mu}}$ become lower as $\dot{\phi}$ increases. The following specific features of the level crossing scheme are worth noticing.

e) If $\dot{\phi}>0$, a resonance in the $\nu_{\mu L} \leftrightarrow \bar{\nu}_{\mu R}$ channel appears for $N>0$ (Fig. 2e). If the adiabaticity condition is fulfilled for this resonance, $\nu_{e L}$ can undergo a transition into $\nu_{\mu L}$ through the two-step resonant process of $\nu_{e L} \rightarrow \bar{\nu}_{\mu R}$ and $\bar{\nu}_{\mu R} \rightarrow \nu_{\mu L}$, imitating the flavour conversion $\nu_{e L} \rightarrow \nu_{\mu L}$.

f) For $\dot{\phi}=\dot{\phi}_{2 m}$, where

$$
\dot{\phi}_{2 m} \equiv 4 c_{2} \delta \frac{r}{1+r},
$$

(Fig. 2f) the resonances in the three channels $\nu_{e L} \leftrightarrow \nu_{\mu L}, \bar{\nu}_{\mu R} \leftrightarrow \nu_{\mu L}$, and $\bar{\nu}_{\mu R} \leftrightarrow \nu_{e L}$ merge in the point $N=N_{f}$.

g) When $\dot{\phi}>\dot{\phi}_{2 m}$, permutation of the flavour and the spin-flavour resonances occurs, so that now $N_{f}>N_{s f}$ in contrast with the case of nontwisting magnetic field. Therefore, if a $\nu_{e L}$ moves from sufficiently high to low density regions and the adiabaticity condition is fulfilled in the flavour resonance, it will be transformed into $\nu_{\mu L}$ rather than $\bar{\nu}_{\mu R}$.

h) For $\dot{\phi}>2 c_{2} \delta$ (Fig. 2h) the spin-flavour resonance disappears if $N>0$ (in this case $\left.N_{s f}<0\right)$. Thus, for $N>0$ only two resonances exist: in the $\nu_{e L} \leftrightarrow \nu_{\mu L}$ and $\nu_{\mu L} \leftrightarrow \bar{\nu}_{\mu R}$ transitions, the latter occurring at larger density. For sufficiently large positive values of $\dot{\phi}$ the $\bar{\nu}_{e R}$ state decouples from the rest of the system.

Let us consider next briefly the transitions of $\bar{\nu}_{e R}$. The most interesting effect is the $\bar{\nu}_{e R} \rightarrow \nu_{e L}$ conversion which can take place for negative values of $\dot{\phi}$ (see Figs. 2a-2d). It can proceed as a direct resonant conversion (Figs. 2a,b), or as a two-step process $\bar{\nu}_{e R} \rightarrow \nu_{\mu L} \rightarrow$ $\nu_{e L}$ (Fig. 2d). In addition, $\bar{\nu}_{e R} \rightarrow \nu_{e L}$ conversion is possible in the case of merging of three resonances (Fig. 2c). This may have important implications for the neutrinos emitted in collapsing stars.

Finally, let us discuss the interesting special case of $r=1$. As it follows from eq. (2), it 
corresponds to propagation of neutrinos in an isotopically neutral medium, $n_{e}=n_{p}=n_{n}$, and can be realized in wide density regions in the supernovae. For $r=1$ the matter density term $(N)$ no longer enters the resonant conditions for the spin-flavour conversions (eqs. (6) and (7)), which take the form:

$$
\dot{\phi}= \pm 2 c_{2} \delta
$$

Thus, the spin-flavour conversions in the case under discussion can take place only in rotating magnetic fields. Further, for $r=1$ the dependences on $N$ of the $\bar{\nu}_{e R}$ and $\nu_{\mu L}$ energy levels, as well as those of the $\nu_{e L}$ and $\bar{\nu}_{\mu R}$ energy levels, are the same: the energy levels $H_{\alpha}$ as functions of $N$ form two pairs of parallel lines (Fig. 3). For $\dot{\phi}=-\dot{\phi}_{m}=-2 c_{2} \delta$, merging of two pairs of resonances, namely, of those in the channels $\nu_{e L} \leftrightarrow \nu_{\mu L}$ and $\nu_{e L} \leftrightarrow \bar{\nu}_{e R}$, and in the channels $\bar{\nu}_{\mu R} \leftrightarrow \nu_{\mu L}$ and $\bar{\nu}_{e R} \leftrightarrow \bar{\nu}_{\mu R}$, takes place. Moreover, the levels of $\nu_{\mu L}$ and $\bar{\nu}_{e R}$ coincide now. This means that the $\bar{\nu}_{e R} \leftrightarrow \nu_{\mu L}$ precession will proceed with practically maximal amplitude for any value of $N$, except in the vicinity of the triple resonance points. For $\dot{\phi}=+2 c_{2} \delta$ the levels of $\bar{\nu}_{\mu R}$ and $\nu_{e L}$ merge, and now the $\nu_{e L} \leftrightarrow \bar{\nu}_{\mu R}$ precession will proceed with maximal amplitude.

One can perform similar analysis in the case $c_{2} \delta<0$. The results can be obtained formally from those derived above for $c_{2} \delta>0$ (see a) - h), etc.) by replacing $c_{2} \delta$ by $-c_{2} \delta$, and by interchanging $\nu_{l L}$ and $\bar{\nu}_{l R}$. For instance, if $\dot{\phi}<0$ there will be again a resonance in the transition $\nu_{e L} \leftrightarrow \bar{\nu}_{e R}$, and for $\dot{\phi}=\dot{\phi}_{1 m}=4 c_{2} \delta /(1+r)$ three resonances, this time in the channels $\bar{\nu}_{e R} \leftrightarrow \bar{\nu}_{\mu R}, \nu_{e L} \leftrightarrow \bar{\nu}_{e R}$ and $\nu_{e L} \leftrightarrow \bar{\nu}_{\mu R}$, will merge in one point $\left(N=N_{\bar{f}}=N_{e \bar{e}}=N_{s f}=-2 c_{2} \delta /(1+\mathrm{r})\right)$.

As we see, an electron neutrino propagating in matter and magnetic field can be transformed into any other state of the neutrino system. The neutrino final state will represent, in general, a mixture of the states of the four neutrinos: $\nu_{e L}, \bar{\nu}_{e R}, \nu_{\mu L}$, and $\bar{\nu}_{\mu R}$. Their relative admixtures in the final state are determined by i) the density $N_{0}$ at which the neutrinos are produced, ii) the order of the different resonances crossed by the neutrinos, iii) the degree of overlapping of different resonances, and iv) by the degree to which the adiabaticity condition 
is fulfilled in each of the resonances. Magnetic field rotation can change the order of the resonances and also can indirectly influence the relevant adiabaticity conditions. In the case of nonuniform field rotation $\ddot{\phi}$ enters the adiabaticity conditions directly [12, 13]. Magnetic field rotation can change significantly the composition of the final neutrino state. The most interesting new effects from a phenomenological point of view are the $\nu_{e L} \leftrightarrow \bar{\nu}_{e R}$ transitions and the merging of different resonances; we will discuss these effects quantitatively in the next two sections.

\section{$4 \nu-\bar{\nu}$ Resonances}

Let us consider the $\nu_{e L} \leftrightarrow \bar{\nu}_{e R}$ transitions in the vicinity of the $\nu_{e L}-\bar{\nu}_{e R}$ level crossing. As we have mentioned earlier, to lowest order of the perturbation theory in the interactions described by $H$ there is no $\nu_{e L}-\bar{\nu}_{e R}$ mixing. The latter arises as a result of the interplay of the two types of mixing present in $H$ in eq. (3): the flavour mixing and the mixing generated by the magnetic moment interaction with the magnetic field. More specifically, according to eq. (3) the transition $\nu_{e L} \rightarrow \bar{\nu}_{e R}$ can proceed in two ways:

$$
\begin{gathered}
\nu_{e L} \longrightarrow \nu_{\mu L} \longrightarrow \bar{\nu}_{e R}, \\
\nu_{e L} \longrightarrow \bar{\nu}_{\mu R} \longrightarrow \bar{\nu}_{e R},
\end{gathered}
$$

i.e. via $\nu_{\mu L}$ or $\bar{\nu}_{\mu R}$ in the intermediate state. In (19) the first transition is due to the flavour mixing $\left(s_{2} \delta\right)$ and the second is induced by the interaction with the magnetic field $\left(\mu B_{\perp}\right)$, and vice versa in (20). Since the system of evolution equations (3) formally coincides with the Schroedinger equation, one can make use of the perturbation theory to get the following amplitudes of the processes (19) and (20):

$$
\begin{aligned}
& M_{1}=-s_{2} \delta \mu B_{\perp} P\left(\nu_{\mu L}\right), \\
& M_{2}=\mu B_{\perp} s_{2} \delta P\left(\bar{\nu}_{\mu R}\right) .
\end{aligned}
$$


Here $P\left(\nu_{\mu L}\right)$ and $P\left(\bar{\nu}_{\mu R}\right)$ are the propagators of the system with $\nu_{\mu L}\left(\bar{\nu}_{\mu R}\right)$ in the intermediate state:

$$
P\left(\nu_{\mu L}\left(\bar{\nu}_{\mu R}\right)\right)=\left(H_{e}-H_{\mu(\bar{\mu})}\right)^{-1} .
$$

The opposite signs in eqs. (21) and (22) are related to the fact that the matrix of Majorana magnetic moments of neutrinos is antisymmetric. Evidently, for $P\left(\nu_{\mu L}\right)=P\left(\bar{\nu}_{\mu R}\right)$ a complete compensation between the two matrix elements takes place and the $\nu_{e L} \rightarrow \bar{\nu}_{e R}$ transition is not possible even in the second order of the perturbation theory. Since the selfenergies of $\nu_{\mu L}$ and $\bar{\nu}_{\mu R}$ are different in matter, the cancellation between the two amplitudes (21) and (22) is not complete. Direct calculation in the second order of perturbation theory yields the following amplitude of the $\nu_{e L} \rightarrow \bar{\nu}_{e R}$ transition:

$$
M\left(\nu_{e L} \rightarrow \bar{\nu}_{e R}\right)=M_{1}+M_{2}=\frac{2 s_{2} \delta \mu B_{\perp}(r N-\dot{\phi} / 2)}{\left[2 c_{2} \delta-(1+r) N\right]\left[2 c_{2} \delta-\dot{\phi}-(1-r) N\right]}
$$

Eq. (24) defines an effective coupling which mixes $\nu_{e L}$ and $\bar{\nu}_{e R}$, or in other words, a nondiagonal $\nu_{e L}-\bar{\nu}_{e R}$ term in the higher order effective Hamiltonian of the neutrino system.

From eq. (24) there follow several qualitative conclusions. i) The $\nu_{e L}-\bar{\nu}_{e R}$ mixing can exist even in the absence of magnetic field rotation [6, 15]. ii) Since $r N \sim n_{n}$, in a nonrotating field the $\nu_{e L}-\bar{\nu}_{e R}$ mixing vanishes when the neutron concentration is zero [15]. iii) For sufficiently small $n_{n}$, the $\nu_{e L}-\bar{\nu}_{e R}$ mixing is induced by the magnetic field rotation. iv) The $\nu_{e L}-\bar{\nu}_{e R}$ mixing is a function of $\dot{\phi}$, and depending on the sign of $\dot{\phi}$ (i.e. on the direction of the field rotation) a twisting magnetic field can either increase or reduce the $\nu_{e L}-\bar{\nu}_{e R}$ mixing, and consequently can enhance or suppress the probability of the $\nu_{e L} \rightarrow \bar{\nu}_{e R}$ transition. In particular, in the $\nu_{\mu L}-\bar{\nu}_{\mu R}$ resonance point, where $\dot{\phi}=2 r N$, one has $M=0$.

In order to study the properties of the $\nu_{e L} \rightarrow \bar{\nu}_{e R}$ resonant conversion one has to solve, in general, the system of neutrino evolution equations (3). However, in some physically interesting cases the $\nu_{e L}-\bar{\nu}_{e R}$ system decouples from $\nu_{\mu L}$ and $\bar{\nu}_{\mu R}$ and its evolution can be analyzed separately. This is realized when the $\nu_{e L} \leftrightarrow \bar{\nu}_{e R}$ conversion resonance is sufficiently well separated both in density and in energy from the resonances associated with the $\nu_{e L} \leftrightarrow$ $\nu_{\mu L}$ and $\nu_{e L} \leftrightarrow \bar{\nu}_{\mu R}$ transitions. In other words, the $\nu_{e L}-\bar{\nu}_{e R}$ resonance should not overlap 
with the $\nu_{e L}-\nu_{\mu L}$ and $\nu_{e L}-\bar{\nu}_{\mu R}$ resonances, and the mixing of $\nu_{e L}$ and $\bar{\nu}_{e R}$ with $\nu_{\mu L}$ and $\bar{\nu}_{\mu R}$ should be sufficiently small.

There are altogether four general conditions ensuring the decoupling of the $\nu_{e L}-\bar{\nu}_{e R}$ system from $\nu_{\mu L}$ and $\bar{\nu}_{\mu R}:\left|H_{\mu}-H_{e}\right| \gg 2\left|H_{\mu e}\right|,\left|H_{\bar{\mu}}-H_{e}\right| \gg 2\left|H_{\bar{\mu} e}\right|,\left|H_{\mu}-H_{\bar{e}}\right| \gg 2\left|H_{\mu \bar{e}}\right|$, and $\left|H_{\bar{\mu}}-H_{\bar{e}}\right| \gg 2\left|H_{\bar{\mu} \bar{e}}\right|$. Using the effective Hamiltonian $\left(H-H_{e} \cdot I\right)$, one obtains in the "vicinity" of the $\nu_{e L}-\bar{\nu}_{e R}$ resonance, where $\left|c_{2} \delta\right| \gg|N+\dot{\phi} / 2|$,

$$
\left|H_{\mu}\right|,\left|H_{\bar{\mu}}\right| \gg\left|2 s_{2} \delta\right|, 2 \mu B_{\perp} .
$$

The conditions for nonoverlapping of the resonances in density scale can be written as

$$
\begin{array}{r}
\left|N_{e \bar{e}}-N_{f(\bar{f})}\right| \gtrsim 2 \tan 2 \theta_{0} N_{f(\bar{f})}, \\
\left|N_{e \bar{e}}-N_{s f(\overline{s f})}\right| \gtrsim 2\left(\mu B_{\perp} /\left|c_{2} \delta \pm \dot{\phi} / 2\right|\right) N_{s f(\overline{s f})},
\end{array}
$$

where $\tan 2 \theta_{0} N_{f(\bar{f})}$ and $\left(\mu B_{\perp} /\left|c_{2} \delta \pm \dot{\phi} / 2\right|\right) N_{s f(\overline{s f})}$, are essentially the half-widths of the MSW and the spin-flavour resonances, the width of the $\nu_{e L}-\bar{\nu}_{e R}$ resonance being considerably smaller. It is easy to show that (26) and (27) are always satisfied when inequalities (25) hold. In a medium with $n_{e}>n_{n}$ conditions (26) and (27) can be realized for $\dot{\phi}<0$ and, e.g. $c_{2} \delta>0$ if, for instance, $2 c_{2} \delta \ll|\dot{\phi}|$, or if $2 c_{2} \delta \gg|\dot{\phi}|$. In the first case for $r \cong 1$ we have (see Fig. 2d) $N_{f} \ll N_{e \bar{e}} \ll N_{s f}$, while in the second the inequalities $N_{e \bar{e}} \ll N_{f}, N_{s f}$ (see Fig. 2a) hold. If in the second case, e.g., $\Delta m^{2}$ is sufficiently large, only the resonance condition (8) can be fulfilled, and so only the $\nu_{e L} \rightarrow \bar{\nu}_{e R}$ conversion can be resonantly enhanced.

If conditions (25) hold, one can block-diagonalize the evolution matrix in eq. (3). The resulting $2 \times 2$ submatrix governing the evolution of the $\nu_{e L}-\bar{\nu}_{e R}$ system $\square$ takes the form:

$$
\left(\begin{array}{cc}
0 & 2 \mu B_{\perp} s_{2} \delta \beta \\
2 \mu B_{\perp} s_{2} \delta \beta & -2 N-\dot{\phi}+2\left(s_{2}^{2} \delta^{2}-\mu^{2} B_{\perp}^{2}\right) \beta
\end{array}\right)
$$

\footnotetext{
${ }^{1}$ In fact, this matrix describes the evolution of $\nu_{e}^{\prime}$ and $\bar{\nu}_{e}^{\prime}$ states obtained as a result of blockdiagonalization of the initial Hamiltonian, but they coincide with $\nu_{e}$ and $\bar{\nu}_{e}$ up to small corrections of the order of $2 \sqrt{\mu^{2} B_{\perp}^{2}+s_{2}^{2} \delta^{2}} /\left|-r N+\dot{\phi} / 2 \pm 2 c_{2} \delta\right|$.
} 
The nondiagonal term, $\bar{H}_{e \bar{e}}$, in the evolution matrix (28) 1 coincides with the effective $\nu_{e L}-\bar{\nu}_{e R}$ mixing parameter obtained earlier in the second order of the perturbation theory, eq. (24). In the approximation used the parameter $\beta$ can be written as

$$
\beta \equiv \frac{-r N+\dot{\phi} / 2}{\left[(-r N+\dot{\phi} / 2)^{2}-\left(2 c_{2} \delta\right)^{2}\right]} .
$$

The resonance condition following from $(28)$ is

$$
N+\dot{\phi} / 2=\left(s_{2}^{2} \delta^{2}-\mu^{2} B_{\perp}^{2}\right) \beta
$$

It is more accurate than the previously found condition, eq. (8), but the correction is small. For $\mu B_{\perp}=s_{2} \delta$ the two conditions coincide. The $\nu_{e L}-\bar{\nu}_{e R}$ mixing angle in matter, corresponding to $(28)$ is given by:

$$
\tan 2 \tilde{\theta}=-\frac{2 \mu B_{\perp} s_{2} \delta \beta}{N+\dot{\phi} / 2-\left(s_{2}^{2} \delta^{2}-\mu^{2} B_{\perp}^{2}\right) \beta}
$$

Let us consider now in greater detail the nondiagonal element $\bar{H}_{e \bar{e}}$ of the $\nu_{e L}-\bar{\nu}_{e R}$ evolution matrix (28). This element determines the level splitting at the $\nu_{e L}-\bar{\nu}_{e R}$ resonance, as well as the degree of adiabaticity of the $\nu_{e L} \leftrightarrow \bar{\nu}_{e R}$ transition. It can be represented as

$$
\bar{H}_{e \bar{e}}=-s_{2} \delta \mu B_{\perp} \frac{H_{\mu}-H_{\bar{\mu}}}{H_{\mu} H_{\bar{\mu}}},
$$

and it follows from eq. (32) that under the conditions ensuring the validity of the evolution matrix (28), the $\nu_{e L}-\bar{\nu}_{e R}$ mixing is always smaller than each of the generic first order mixings $s_{2} \delta$ and $\mu B_{\perp}$. If one of the two elements $H_{\mu}$ and $H_{\bar{\mu}}$ is much bigger than the other, $\bar{H}_{e \bar{e}}$ will be suppressed by the smaller of the two: e.g., if $\left|H_{\bar{\mu}}\right| \gg\left|H_{\mu}\right|$, we have $\bar{H}_{e \bar{e}} \sim-s_{2} \delta \mu B_{\perp} / H_{\mu}$. At the $\nu_{e L} \leftrightarrow \bar{\nu}_{e R}$ resonance point (where $N=N_{e \bar{e}}=-\dot{\phi} / 2$ ) $\bar{H}_{e \bar{e}}$ can be written as:

$$
\begin{gathered}
\bar{H}_{e \bar{e}}^{r e s}=-s_{2} \delta \mu B_{\perp} \frac{2 N_{e \bar{e}}(1+r)}{\left(2 c_{2} \delta\right)^{2}-(1+r)^{2} N_{e \bar{e}}^{2}}=\mp t_{2} \mu B_{\perp} \frac{N_{e \bar{e}} / N_{f(\bar{f})}}{1-\left(N_{e \bar{e}} / N_{f(\bar{f})}\right)^{2}}= \\
=-s_{2} \delta \mu B_{\perp} \frac{2(1+r) /(1-r)^{2}}{N_{e \bar{e}}\left(1-N_{s f} / N_{e \bar{e}}\right)\left(1-N_{\bar{s} f} / N_{e \bar{e}}\right)},
\end{gathered}
$$

\footnotetext{
${ }^{2}$ We have again subtracted a matrix proportional to the unit matrix in such a way that the upper diagonal element in the $\nu_{e L}-\bar{\nu}_{e R}$ evolution matrix (28) thus derived is zero.
} 
where $t_{2} \equiv \tan 2 \theta_{0}$, and $N_{f(\bar{f})}$ and $N_{s f(\bar{s} f)}$ are the MSW and the spin-flavour resonance densities determined in eqs. (11) and (12). It follows from eq. (33) that the $\nu_{e L}-\bar{\nu}_{e R}$ mixing term at the resonance increases when $N_{e \bar{e}}$ approaches $N_{f}$, i.e when the $\nu_{e L}-\bar{\nu}_{e R}$ resonance approaches the MSW resonance 3 . However, in the approximation we use, which implies a sufficiently good separation between the different resonances, $N_{e \bar{e}}$ cannot be very close to $N_{f}$. Using criteria (25) and choosing $N_{e \bar{e}}=N_{f} \pm 2 t_{2} N_{f}$ in eq. (33) one obtains for the allowed maximal value of $\bar{H}_{e \bar{e}}$,

$$
\bar{H}_{e \bar{e}}^{r e s} \cong t_{2} \mu B_{\perp} \frac{1 \pm 2 t_{2}}{1-\left(1 \pm 2 t_{2}\right)^{2}}
$$

and for small values of $t_{2}\left(2\left|t_{2}\right| \ll 1\right)$ one finds: $\left|\bar{H}_{e \bar{e}}^{r e s}\right| \cong \frac{1}{4} \mu B_{\perp}$. Thus, the "maximal" value of $\left|\bar{H}_{e \bar{e}}^{r e s}\right|$ in the case considered is only by a factor of 4 smaller than the spin-flavour mixing element in $H$.

From (33) it follows that $\left|\bar{H}_{e \bar{e}}^{r e s}\right|$ increases also when $N_{e \bar{e}}$ tends to $N_{\bar{s} f}$, i.e. when the $\nu_{e L}-\bar{\nu}_{e R}$ resonance approaches the $\bar{\nu}_{e R}-\nu_{\mu L}$ resonance. The "maximal" value of $\left|\bar{H}_{e \bar{e}}^{r e s}\right|$ compatible with constraint (27) in this case is $\frac{1}{4}\left|s_{2} \delta\right|$ and is reached for $\mu B_{\perp} /\left(c_{2} \delta\right) \ll 1$.

The mixing parameter $\bar{H}_{e \bar{e}}^{r e s}$ enters the expression for the adiabaticity parameter characterizing the $\nu_{e L} \leftrightarrow \bar{\nu}_{e R}$ transitions: $\kappa_{R}=\left(2 \bar{H}_{e \bar{e}}^{r e s}\right)^{2} / \dot{H}_{\bar{e}, \text { res }}$, where $\dot{H}_{\bar{e}, \text { res }}$ is the derivative of $H_{\bar{e}}$ at the resonance, $H_{\bar{e}}$ being the $\nu_{e L}-\bar{\nu}_{e R}$ level splitting (see eq. (10)). Using the lowest order expression for $H_{\bar{e}}$, on finds:

$$
\kappa_{R}=t_{2}^{2}\left(\mu B_{\perp}\right)^{2} \frac{L_{e \bar{e}}}{N_{e \bar{e}}} \frac{2\left(N_{e \bar{e}} / N_{f}\right)^{2}}{\left[1-\left(N_{e \bar{e}} / N_{f}\right)^{2}\right]^{2}} .
$$

Here $L_{e \bar{e}}=N_{e \bar{e}} /\left|(\dot{N}+\ddot{\phi} / 2)_{r e s}\right|$, where the value of $(\dot{N}+\ddot{\phi} / 2)$ is taken at the resonance point. For $\kappa_{R} \gg 1$ the $\nu_{e L} \rightarrow \bar{\nu}_{e R}$ transition is adiabatic and $\nu_{e L}$ can be completely converted into $\bar{\nu}_{e R}$. Again, when $N_{e \bar{e}}$ approaches $N_{f}$ (and/or $N_{\bar{s} f}$ ) the adiabaticity of the $\nu_{e L} \rightarrow \bar{\nu}_{e R}$ transition improves.

\footnotetext{
${ }^{3}$ We assume here that $c_{2} \delta>0$.
} 


\section{Triple Resonance. Neutrino - Antineutrino Oscilla- tions.}

We shall analyze here the case when resonances merge, which is opposite to the one discussed in Section 4. The mutual influence of the different neutrino transitions becomes very strong in this case.

If $\dot{\phi}=\dot{\phi}_{1 m}<0$ (see Section 3, eq. (16)), three level crossing points, namely, the $\nu_{e L}-\nu_{\mu L}, \bar{\nu}_{e R}-\nu_{\mu L}$, and $\bar{\nu}_{e R}-\nu_{e L}$, merge at $N=N_{f}$. The condition of merging depends on the neutrino energy $E$; for fixed $E$ the resonances merge at definite value of $\dot{\phi}$. If neutrinos possess continuous energy spectrum, the merging will occur for a given $\dot{\phi}<0$ at definite value of the energy, $E_{m}$ :

$$
E_{m}=-\frac{c_{2} \Delta m^{2}}{(1+r) \dot{\phi}} .
$$

Thus, the specific phenomena which take place at the merging point will have a resonance character as functions of energy.

Suppose $\dot{\phi}=\dot{\phi}_{1 m}$ and consider the dynamics of the neutrino system in the vicinity of the merging point which we shall call also point of the triple resonance. Now three neutrino states are participating in the transitions and only one state, that of $\bar{\nu}_{\mu R}$, can decouple in the triple resonance region. One has for this state

$$
H_{\bar{\mu}}=-(1-r) N+\frac{2 c_{2} \delta(3+r)}{1+r}
$$

If $s_{2} \delta, \mu B_{\perp} \ll H_{\bar{\mu}}$ (which implies that $s_{2} \ll 1$ and $\mu B_{\perp} \ll 4 c_{2} \delta$ ), it is possible to perform a $3-1$ block-diagonalization of the neutrino evolution matrix in the triple resonance region. This leads to the following effective Hamiltonian for the $\left(\nu_{e L}, \bar{\nu}_{e R}, \nu_{\mu L}\right)$ system:

$$
H^{\prime}=\left(\begin{array}{ccc}
-\frac{\left(\mu B_{\perp}\right)^{2}}{H_{\bar{\mu}}} & -\epsilon & s_{2} \delta \\
-\epsilon & -2 N+\frac{4 c_{2} \delta}{1+r}-\frac{\left(s_{2} \delta\right)^{2}}{H_{\bar{\mu}}} & -\mu B_{\perp} \\
s_{2} \delta & -\mu B_{\perp} & -N(1+r)+2 c_{2} \delta
\end{array}\right)
$$


where

$$
\epsilon=\frac{\left(s_{2} \delta\right)\left(\mu B_{\perp}\right)}{H_{\bar{\mu}}}
$$

corresponds to a small direct $\nu_{e L}-\bar{\nu}_{e R}$ mixing of the second order, generated by the propagation only of $\bar{\nu}_{\mu R}$. As we shall see, the mixing induced by the $\nu_{\mu L}$-exchange is resonantly enhanced and turns out to be of the order of the generic mixings. The diagonal elements also acquire corrections due to the propagation of $\bar{\nu}_{\mu R}$ which are written explicitly in (38). They modify condition (36) and the density at the triple crossing point by terms $\sim \epsilon$.

Let us find the level splitting and the mixing in the neutrino system described by the Hamiltonian (38), in the merging point. The eigenvalues of the Hamiltonian (38) read:

$$
H_{1} \cong f-\frac{\epsilon^{\prime}}{2}, H_{2} \cong-f-\frac{\epsilon^{\prime}}{2}, H_{3} \cong \epsilon^{\prime} .
$$

Here

$$
f=\sqrt{\left(\mu B_{\perp}\right)^{2}+\left(s_{2} \delta\right)^{2}}
$$

and

$$
\epsilon^{\prime}=-\epsilon \sin 2 \omega
$$

where

$$
\tan \omega=\frac{s_{2} \delta}{\mu B_{\perp}}
$$

is the ratio of the leading order flavour and spin-flavour mixing terms. The parameter $f$ determines the level splitting in the leading order (i.e. neglecting the terms $\sim \epsilon$ ): $H_{1}-H_{2}=$ $2 f, H_{1}-H_{3}=H_{3}-H_{2}=f$. Moreover, the flavour and the spin-flavour mixing terms enter $f$ symmetrically. (For comparison, notice that the splitting in the flavour and in the spinflavour resonance points are equal, respectively, to $2 s_{2} \delta$ and $2 \mu B_{\perp}$.) The $H_{1}-H_{2}$ splitting does not depend on the $\bar{\nu}_{\mu R}$-level correction $\epsilon^{\prime}$, while $H_{3}$ shifts towards $H_{1}$ or $H_{2}$ depending on the sign of $\epsilon^{\prime}$.

The orthogonal matrix diagonalizing the Hamiltonian (38) is given to leading (first) 
order correction in $\epsilon / f$ by

$$
S_{m}=\left(\begin{array}{ccc}
\frac{\sin \omega}{\sqrt{2}}+\alpha & \frac{\sin \omega}{\sqrt{2}}-\alpha & \cos \omega \\
-\frac{\cos \omega}{\sqrt{2}}+\gamma & -\frac{\cos \omega}{\sqrt{2}}-\gamma & \sin \omega \\
\frac{1}{\sqrt{2}}-\xi & -\frac{1}{\sqrt{2}}-\xi & -\frac{\epsilon}{f} \cos 2 \omega
\end{array}\right)
$$

where the parameters $\alpha, \gamma$ and $\xi$ are all of the order of $\epsilon / f$ :

$$
\begin{gathered}
\alpha=\frac{1}{\sqrt{2}} \frac{\epsilon}{f} \cos \omega\left(1-\frac{3}{2} \sin ^{2} \omega\right), \\
\gamma=-\frac{1}{\sqrt{2}} \frac{\epsilon}{f} \sin \omega\left(1-\frac{3}{2} \cos ^{2} \omega\right), \\
\xi=\frac{1}{4 \sqrt{2}} \frac{\epsilon}{f} \sin 2 \omega .
\end{gathered}
$$

Consider now the properties of the various neutrino transitions which can take place at the merging point. If the density and the chemical composition of the medium, and the magnetic field (i.e. $N, r, B_{\perp}$ and $\dot{\phi}$ ) do not change along the neutrino trajectory, the propagation of neutrinos has a character of pure oscillations-precessions with constant depths and periods. There are altogether three transition channels, $\nu_{e L} \leftrightarrow \nu_{\mu L}, \bar{\nu}_{e R} \leftrightarrow \nu_{\mu L}$, $\nu_{e L} \leftrightarrow \bar{\nu}_{e R}$, and we shall concentrate on the qualitatively new mode $\nu_{e L} \leftrightarrow \bar{\nu}_{e R}$. Using (40) and (44) we find for the relevant oscillation-precession probability in a medium with $N, B_{\perp}$, and $\dot{\phi}$ having values satisfying the merging condition:

$$
P\left(\nu_{e L} \rightarrow \bar{\nu}_{e R}\right)=\sin ^{2} 2 \omega \sin ^{4} \frac{1}{2} f t+\sin ^{2} 2 \omega \sin ^{2} \frac{3}{4} \epsilon^{\prime} t \cos f t+d \sin \epsilon^{\prime} t \sin f t
$$

where

$$
\sin ^{2} 2 \omega=\frac{4\left(s_{2} \delta\right)^{2}\left(\mu B_{\perp}\right)^{2}}{\left[\left(s_{2} \delta\right)^{2}+\left(\mu B_{\perp}\right)^{2}\right]^{2}}
$$

and $d=O(\epsilon)$. As we have already emphasized, the $\nu_{e L} \leftrightarrow \bar{\nu}_{e R}$ oscillations-precessions can exist only in the presence of both vacuum mixing and magnetic moment interaction. The first term in (48) corresponds to the lowest order, obtained when the term $\epsilon$ in (38) is neglected. The depth of the oscillations, $\sin ^{2} 2 \omega$, does not exhibit any suppression related to the higher order direct $\nu_{e L}-\bar{\nu}_{e R}$ mixing. Moreover, in the symmetric case when the 
vacuum mixing is equal to that induced by the magnetic moment interaction, $s_{2} \delta=\mu B_{\perp}$, the oscillation depth becomes maximal. It should be stressed that this is possible only in twisting magnetic fields. For $s_{2} \delta \neq \mu B_{\perp}$ the oscillation depth is less than unity and it decreases when the difference between $s_{2} \delta$ and $\mu B_{\perp}$ increases.

The $\nu_{e L} \leftrightarrow \bar{\nu}_{e R}$ oscillation-precession length is given by

$$
l_{e \bar{e}}=\frac{2 \pi}{f}=\frac{2 \pi}{\sqrt{\left(s_{2} \delta\right)^{2}+\left(\mu B_{\perp}\right)^{2}}} .
$$

For $\left(s_{2} \delta\right) \ll\left(\mu B_{\perp}\right)$, this length $l_{e \bar{e}}$ is two times bigger than the usual precession length, $l_{p}$ : $l_{e \bar{e}} \cong 2 l_{p}=2 \pi /\left(\mu B_{\perp}\right)$. For $\left(s_{2} \delta\right) \gg\left(\mu B_{\perp}\right)$ it is twice as big as the oscillation length in matter at resonant density, $l_{o s c}: l_{e \bar{e}} \cong 2 l_{o s c}=8 \pi E /\left(\Delta m^{2} \sin 2 \theta_{0}\right)$. In the symmetric case when $\left(s_{2} \delta\right)=\left(\mu B_{\perp}\right)$ one has: $l_{e \bar{e}}=\sqrt{2} l_{o s c}=\sqrt{2} l_{p}$. Finally, note that $P\left(\nu_{e L} \rightarrow \bar{\nu}_{e R}\right)$ depends on the fourth power of $\sin \left(\pi t / l_{e \bar{e}}\right)$ rather than on the second power, as in the usual case.

These features of the $\nu_{e L} \leftrightarrow \bar{\nu}_{e R}$ oscillations are consequences of the facts that there are three levels involved and that the splitting between the levels is determined by $f$. More precisely, according to (44), $\nu_{e L}$ and $\bar{\nu}_{e R}$ are (to leading order) orthogonal combinations of two states: $\nu_{0}$ with energy $H_{3} \cong 0$, and $\nu^{\prime}$ which itself is a maximal mixture of two states with opposite energies $\pm f$. The amplitudes of the $\nu_{0} \rightarrow \nu_{0}$ and $\nu^{\prime} \rightarrow \nu^{\prime}$ transitions are equal, respectively, to 1 and cos $f t$. Their sum in the amplitude of the $\nu_{e L} \rightarrow \bar{\nu}_{e R}$ transition gives $(1-\cos f t)=2 \sin ^{2} \frac{1}{2} f t$. As a consequence, the $\nu_{e L} \rightarrow \bar{\nu}_{e R}$ oscillation probability is proportional to $\sin ^{4} \frac{1}{2} f t$.

The second term in (48) is generated by the direct $\nu_{e L}-\bar{\nu}_{e R}$ mixing in (38). It gives a long-period modulation of the oscillation probability. For the corresponding modulation length, $l_{\text {mod }}$, one finds: $l_{\text {mod }} \cong 4 \pi /\left(3 \epsilon^{\prime}\right) \gg l_{e \bar{e}}$. The first two terms in (48) can also be written as $\frac{1}{4} \sin ^{2} 2 \omega\left(1+\cos ^{2} f t-2 \cos f t \cos \frac{3}{2} \epsilon^{\prime} t\right)$, which implies that the modulation leads to the oscillation depth varying between $\sin ^{2} 2 \omega$ and $\frac{1}{2} \sin ^{2} 2 \omega$.

Let us turn next to the neutrino transitions in the region of the merging point in the case of varying matter density. The efficiencies of the resonant conversions depend on the degree 
of their adiabaticity. In what follows we will neglect the corrections $\sim \epsilon$ due to the $\bar{\nu}_{\mu R}$ level. This can be done, in particular, because the time of propagation in the resonance region $\left(t \sim \Delta r_{r e s}\right)$ is much smaller than the period $\sim 1 / \epsilon$. In addition, as we have established above, the relevant level splittings are determined by $s_{2} \delta$ and $\mu B_{\perp}$, the terms $\sim \epsilon$ giving only small corrections.

Consider first the dependence of the energy levels on $N$ (Fig. 4). Its specific features are determined essentially by the relative magnitude of the vacuum $\left(s_{2} \delta\right)$ and the magnetic moment induced $\left(\mu B_{\perp}\right)$ mixings. In particular, a critical value of the ratio of the two mixing elements exists,

$$
\left(\frac{s_{2} \delta}{\mu B_{\perp}}\right)_{c}=\tan \omega_{c} \equiv \sqrt{\frac{1+r}{1-r}} .
$$

If $\tan \omega=\tan \omega_{c}$, the eigenvalue $H_{3}(N)$ of the Hamiltonian (38) will coincide with the flavour level energy $H_{\mu}(N)$ for all values of $N$ (Fig. 4a):

$$
H_{3}(N)=H_{\mu}(N)=-(1+r) N+2 c_{2} \delta
$$

The dependence on $N$ of the other two eigenvalues of (38) can also be found:

$$
H_{1,2}=\frac{H_{\bar{e}}}{2} \pm \sqrt{\frac{H_{\bar{e}}^{2}}{4}+f^{2}},
$$

where $H_{\bar{e}}$ is defined in eq. (10). There is a simple interpretation of condition (51) and the critical regime of transitions associated with it. Indeed, eq. (51) can be rewritten as

$$
\frac{\left|H_{e}-H_{\mu}\right|}{\left|H_{\mu}-H_{\bar{e}}\right|}=\frac{\left(s_{2} \delta\right)^{2}}{\left(\mu B_{\perp}\right)^{2}}
$$

Now, the mixing, as is well known, leads to a "repulsion" of the levels: the energy splitting between the levels becomes bigger than that without mixing. The larger the mixing and the smaller the splitting between the corresponding flavour levels, the larger the repulsion effect. In our case two states influence the $H_{3}$ level (which coincides with that of $\nu_{\mu L}$ at high densities). Then eq. (54) is nothing else but a balance condition ensuring that the repulsion of the $\nu_{\mu}$ level from the $\nu_{e L}$ and $\bar{\nu}_{e R}$ ones compensate each other for all densities. In other words, the shift of the $H_{3}$ level resulting from the flavour mixing is compensated 
by that stemming from the magnetic-moment induced mixing. Due to this balance the $\nu_{1}$ level does not deviate from the $\nu_{\mu L}$ one and turns out to be a straight line coinciding with $H_{\mu}(N)$.

In the critical case the minimal splittings between all levels happen in the merging point. They are determined by eqs. (40).

The case $\tan \omega<\tan \omega_{c}$ corresponds to relatively weak vacuum mixing. Now the levels of the neutrinos participating in the spin-flavour conversion, i.e. $\bar{\nu}_{e R}$ and $\nu_{\mu L}$, repulse each other stronger than the $\nu_{e L}$ and $\nu_{\mu L}$ levels, and $H_{3}(N)$ will not coincide with $H_{\mu}(N)$ (Fig. 4b). The points of minimal splitting between the eigenvalues are shifted away from the merging point symmetrically to $N_{m} \pm \Delta N_{m}$. Moreover, the splitting itself becomes smaller than $f$. With decreasing the ratio $\left(s_{2} \delta\right) /\left(\mu B_{\perp}\right)$ the shift of the minima from the merging point increases approaching the maximal value

$$
\Delta N_{m}=\frac{\mu B_{\perp}}{\sqrt{2(1+r)}} .
$$

The densities $N_{m} \pm \Delta N_{m}$ correspond to the points at which the $\nu_{e L}$-level crosses the levels of the eigenstates of the Hamiltonian describing the $\left(\bar{\nu}_{e R}, \nu_{\mu L}\right)$ system when $s_{2} \delta=0$ (i.e. when the $\nu_{e L}$-level decouples). The level splitting in these points

$$
\Delta H=2 \sqrt{\frac{2}{3+r}} s_{2} \delta
$$

is determined by the (smaller) flavour mixing.

For $\tan \omega>\tan \omega_{c}$ the vacuum mixing dominates over the magnetic moment mixing. The levels of the neutrinos taking part in the spin-flavour conversion effectively attract each other now (Fig. 4c). Again, there are two points of minimal splitting, and they are shifted with respect to the merging point. When $s_{2} \delta /\left(\mu B_{\perp}\right)$ increases the shift $\Delta N_{m}$ in the points of minimal splitting converges to

$$
\Delta N_{m}=\frac{s_{2} \delta}{\sqrt{2(1-r)}},
$$


and the splitting in these points becomes

$$
\Delta H=2 \sqrt{\frac{2}{3-r}} \mu B_{\perp} .
$$

The shifts (57) can be deduced from the densities in the crossing points between the $\bar{\nu}_{e R}$ level and the levels corresponding to the eigenvalues of the $\left(\nu_{e L}, \nu_{\mu L}\right)$ Hamiltonian when $\mu B_{\perp}=0$ (i.e. when the $\bar{\nu}_{e R}$ level decouples). The splitting is determined only by the magnetic field induced mixing.

The corrections due to the $\bar{\nu}_{\mu R}$ state give rise to small $(\sim \epsilon)$ shifts of the levels and to a breaking of the symmetry of the level-crossing scheme (with respect to the reflections $\left.\left(N-N_{m}\right) \rightarrow-\left(N-N_{m}\right), H_{i} \rightarrow-H_{i}\right)$.

\section{Resonant Conversions in the Merging Region}

Let us discuss further the resonant transitions in the triple resonance region. Now three neutrino levels are involved simultaneously in the relevant transitions and the adiabaticity conditions are more complicated than in the two-neutrino case. One can introduce partial adiabaticity parameters $\kappa^{(i j)}, i, j=1,2,3$, characterizing the "jump" probabilities between given pairs of levels, $H_{i}(N)$ and $H_{j}(N)$. The partial adiabaticity parameter $\kappa^{(i j)}$ can be defined as a ratio of the spatial width of the corresponding resonance region, $\Delta r_{R}^{i j}$, and the oscillation (precession) length at the resonance, $l_{R}^{i j}: \kappa^{(i j)}=\pi \Delta r_{R}^{i j} / l_{R}^{i j}$. The oscillation/precession length $l_{R}^{i j}$ is determined by the minimal level splitting: $l_{R}^{i j} \cong 2 \pi / \Delta H_{i j}^{\min }$, $\Delta H_{i j}^{\min }=\min \Delta H_{i j}(N)=\min \left(H_{i}(N)-H_{j}(N)\right)=H_{i}\left(N_{i j}^{\min }\right)-H_{j}\left(N_{i j}^{\min }\right)$. The width of the resonance $\Delta r_{R}^{i j}$ can be estimated as the distance over which the level splitting becomes by a factor $\sqrt{2}$ bigger than the minimal one:

$$
\Delta r_{R}^{i j} \cong 2\left|(\dot{N})_{r e s}\right|^{-1} \Delta N_{i j},
$$

where $\Delta N_{i j}=\left|N-N_{i j}^{m i n}\right|$ is determined by the condition

$$
\left|\Delta H_{i j}\left(N_{i j}^{m i n} \pm \Delta N_{i j}\right)\right|=\sqrt{2}\left|\Delta H_{i j}^{m i n}\right| .
$$


This definition is equivalent to the definition considered in Section 4 for the two-neutrino case.

An interesting feature of the three level system under discussion is that the $\nu_{3}$ level approaches (as function of $N$ ) the $\nu_{1}$ and $\nu_{2}$ levels not symmetrically with respect to the point of minimal splitting $N_{\text {min }}$, i.e. the way the levels approach each other when $N \rightarrow N_{\min }$ depends on whether $N<N_{\min }$ or $N>N_{\min }$. (This is equivalent to having two different dependences of $N(t)$ on $t$ for $N>N_{\min }$ and $N<N_{\min }$ (see Fig. 4)). In this case one could introduce two adiabaticity parameters for each pair of levels, $\kappa_{a}^{(i j)}=\kappa^{(i j)}\left(N>N_{m i n}^{i j}\right)$, and $\kappa_{b}^{(i j)}=\kappa^{(i j)}\left(N<N_{m i n}^{i j}\right)$, as well as an average adiabaticity parameter $\bar{\kappa}^{(i j)}=\left(\kappa_{a}^{(i j)}+\kappa_{b}^{(i j)}\right) / 2$.

Consider now the resonant transitions for different values of the ratio $\left(s_{2} \delta\right) /\left(\mu B_{\perp}\right)$.

For critical values of the parameters (Fig. 4a) the minimal splittings for all three types of transitions occur at the merging point. The adiabaticity parameter determining the jump probability from the $\nu_{1}$ level (which approaches $\nu_{e L}$ for sufficiently large values of $N$ ) to the $\nu_{3}$ level $\left(\sim \nu_{\mu L}\right)$ is given by

$$
\bar{\kappa}^{(13)}=\sqrt{2} \frac{f^{2}}{1-r^{2}}\left|(\dot{N})_{r e s}\right|^{-1}
$$

(we have used the relations $\Delta H(N) \cong H_{e}-H_{\mu}$ for $N>N_{\min }$, and $\Delta H(N) \cong H_{\bar{e}}-H_{\mu}$ for $N<N_{\min }$, and the fact that $\Delta H_{\min }=f$, in obtaining eq. (61)). The adiabaticity parameter for the transition $\nu_{2} \leftrightarrow \nu_{3}$ coincides with $\bar{\kappa}^{(13)}$. One has for the transition $\nu_{1} \leftrightarrow \nu_{2}$

$$
\kappa^{(12)}=2 \sqrt{2} f^{2}\left|(\dot{N})_{r e s}\right|^{-1}
$$

Note that all adiabaticity parameters are determined by the same splitting parameter $f$, and if $r$ is not too close to 1 , are of the same order. If the adiabaticity conditions for the transitions of $\nu_{1}$ are fulfilled $\left(\bar{\kappa}^{(12)} \gtrsim 1, \kappa^{(13)} \gtrsim 1\right)$, and $\nu_{e L}$ is produced far from the merging point at $N_{0} \gg N_{m}$, it will be almost completely converted into $\bar{\nu}_{e R}$ provided $N_{0}<N_{s f}$. If the adiabaticity is weakly broken, $\nu_{e L}$ will be transformed with large probability into $\bar{\nu}_{e R}$, with rather small probability into $\nu_{\mu L}$, and with even smaller probability will remain 
$\nu_{e L}$. For $\kappa^{(13)} \sim \kappa^{(12)}$ the probabilities of the $\nu_{e L} \rightarrow \nu_{\mu L}$ and $\nu_{e L} \rightarrow \bar{\nu}_{e R}$ transitions will be comparable.

Suppose next that $\nu_{\mu L}$ is produced at $N \gg N_{m}$. Then the neutrino system will be on the $\nu_{3}$ level and in the case of adiabatic change of density (i.e. $\kappa^{(31)} \gtrsim 1$ ) will end up as $\nu_{\mu L}$ at $N=0$. However, the flavour content of the $\nu_{3}$ state (which coincides at high densities with $\nu_{\mu L}$ ) changes with the variation of density:

$$
\mid \nu_{3}>=\left\{\mu B_{\perp}\left|\nu_{e L}>+s_{2} \delta\right| \bar{\nu}_{e R}>-(1+r)\left(N-N_{m}\right) \frac{\mu B_{\perp}}{s_{2} \delta} \mid \nu_{\mu L}>\right\} R,
$$

where $\mu B_{\perp}$ and $s_{2} \delta$ obey eq. (51) and $R(N)$ is a normalization factor. Note that the relative admixtures of the $\nu_{e L}$ and the $\bar{\nu}_{e R}$ states are fixed, and the $\nu_{\mu L}$ admixture changes with $N$. In the merging point the latter is zero, i.e. the $\nu_{3}$ state is composed only of $\nu_{e L}$ and $\bar{\nu}_{e R}$.

For the noncritical values of $s_{2} \delta /\left(\mu B_{\perp}\right)$ (Figs. 4b,c) the results in the case of adiabatic transitions are the same $\left(\nu_{e L} \rightarrow \bar{\nu}_{e R}\right.$, etc. $)$, but the types of the transitions change when the adiabaticity conditions do not hold. Now besides the merging point there are two other points of minimal splitting, at $N=N_{1}$ and at $N=N_{2}$. The splittings and the adiabaticity conditions in these two points are determined by the smallest mixing (flavour or magnetic moment induced) rather than by $f$. Thus, the "jumps" are more probable in the points $N_{1}$ and $N_{2}$ than in the merging point itself. Moreover, all conditions governing the neutrino transitions in the regions of $N_{1}$ and $N_{2}$ are identical (up to corrections $\sim \epsilon$ ). Therefore the splittings, the adiabaticity parameters, and the amplitudes of the transitions are the same in $N_{1}$ and $N_{2}$ (if, of course, $|\dot{N}|$ has the same value in the two points).

In the case of relatively weak vacuum mixing $\left(s_{2} \delta<\mu B_{\perp}\right)$ the $\nu_{e L}$ trajectory crosses both minimal splitting regions. The scheme of the transitions for $\nu_{e L}$ is similar to that in the critical regime: if the adiabaticity is only weakly broken, $\nu_{e L}$ will be transformed mainly into $\bar{\nu}_{e R}$, and with a smaller probability into $\nu_{\mu L}$ (the probability of $\nu_{e L}$ to remain $\nu_{e L}$ being strongly suppressed). In the case of strong adiabaticity violation in the points $N_{1,2}, \nu_{e L}$ remains with large probability $\nu_{e L}$ and only small and equal admixtures of $\bar{\nu}_{e R}$ and $\nu_{\mu L}$ in the final state will appear. Now the two-step process $\nu_{e L} \rightarrow \nu_{\mu L} \rightarrow \nu_{e L}$, whose probability 
depends on the adiabaticity in the two minimal splitting points, can be more efficient than the direct "jump" $\nu_{e L} \rightarrow \nu_{e L}$ (from the level $\nu_{1}$ to the level $\nu_{2}$ ) which depends on the big splitting $2 f$.

The neutrino transitions possess interesting features also when the initial state is $\nu_{\mu L}$. Its trajectory crosses the two minimal splitting regions as well. For sufficiently weak violation of the adiabaticity $\nu_{\mu L}$ will be transformed into $\nu_{e L}$ and $\bar{\nu}_{e R}$ with rather small and comparable probabilities. In the case of strong adiabaticity violation $\nu_{\mu L}$ will be converted mainly into $\bar{\nu}_{e R}$. Moreover, the stronger the violation of the adiabaticity of the transition, the smaller the $\nu_{\mu L}$ survival probability, in contrast to the behaviour of the $\nu_{\mu L}$ survival probability in the usual two-neutrino case.

Suppose further that the vacuum mixing is relatively strong $\left(s_{2} \delta>\mu B_{\perp}\right.$, Fig. $\left.4 \mathrm{c}\right)$. Now the conditions in the two minimal splitting points, governing the conversions of $\nu_{e L}$ produced at $N_{0} \gg N_{m}$, can be different from those in the previously considered case. Indeed, at $N \cong N_{1}$ the splittings between the $\nu_{1}$ level and the other two levels turn out to be appreciable, so that the passing through the first minimum splitting region at $N=N_{1}$ will not influence the $\nu_{e L}$ state considerably. Thus, neutrinos produced as $\nu_{e L}$ will cross only one minimal splitting region (at $N=N_{2}<N_{m}$ ) on their way from high to low density domains and in the case of strong adiabaticity violation in this region they will be transformed into $\nu_{\mu L}$. The behaviour of the neutrino system in the case of $\bar{\nu}_{e R}$ in the initial state can be formally obtained from that with $\nu_{e L}$ in the initial state by interchanging the configurations shown on Figs. 4b and 4c.

Let us comment finally on the two mechanisms of $\nu_{e L} \rightarrow \bar{\nu}_{e R}$ transitions. The $\nu_{e L} \rightarrow \bar{\nu}_{e R}$ resonant conversion due to the direct (second order) $\nu_{e L}-\bar{\nu}_{e R}$ mixing and the two-step conversion involving two resonant transitions $\nu_{e L} \rightarrow \nu_{\mu L}$ and $\nu_{\mu L} \rightarrow \bar{\nu}_{e R}$ coincide (are indistinguishable) at the merging point. One can distinguish the two different mechanisms of $\nu_{e L} \rightarrow \bar{\nu}_{e R}$ conversion if the resonance regions of the $\nu_{e L} \rightarrow \bar{\nu}_{e R}, \nu_{e L} \rightarrow \nu_{\mu L}$, and $\nu_{\mu L} \rightarrow \bar{\nu}_{e R}$ transitions are sufficiently well separated. 
The direct resonant conversion takes place in one resonance region, the corresponding resonance condition does not depend (in the leading approximation) on the neutrino energy, and the energy dependence of the conversion probability follows from the energy dependence of the relevant adiabaticity parameter. The level splitting at the resonance point is determined by the product of the flavour and the magnetic field induced mixing elements and typically is rather small. In contrast, in the case of the two-step conversion two resonant transitions take place in two spatial regions which are separated by macroscopic distance. A real $\nu_{\mu L}$ propagates between the two resonant points. Both relevant resonance conditions depend on the neutrino energy. In each of the two resonance points the mixing is generic, and the splitting is generated by first order mixing. There are two adiabaticity parameters (and conditions) which depend on the derivative of $N$ in two different regions of the neutrino trajectory.

Given similar conditions in the resonance regions of the three transitions $\nu_{e L} \rightarrow \bar{\nu}_{e R}$, $\nu_{e L} \rightarrow \nu_{\mu L}$, and $\nu_{\mu L} \rightarrow \bar{\nu}_{e R}$, the two-step $\nu_{e L} \rightarrow \bar{\nu}_{e R}$ conversion will be more efficient because the adiabaticity conditions will be fulfilled better for each of the two transitions involved than for the direct conversion. Nevertheless, under certain conditions the direct conversion can be the dominant mechanism for the $\nu_{e L} \rightarrow \bar{\nu}_{e R}$ transitions. This can be the case in the situations corresponding to the energy level scheme of Fig. 2d provided $N_{e \bar{e}}<N_{0}<N_{s f}$. This can also happen if $N_{0}$ satisfies the conditions $N_{e \bar{e}}<N_{0}<N_{f}$ (Fig. 2a) and if, in addition, the spin-flavour transition $\bar{\nu}_{e R} \rightarrow \nu_{\mu L}$ is non-adiabatic (Fig. 2b). The direct $\nu_{e L} \rightarrow \bar{\nu}_{e R}$ transitions can also be efficient if $\bar{\nu}_{e R}$ is produced in the initial state and propagates from $N_{0}>N_{e \bar{e}}$ to $N \rightarrow 0$. In all these cases the adiabaticity condition for the direct $\nu_{e L} \rightarrow \bar{\nu}_{e R}$ conversion can be fulfilled, in particular, due to relatively small value of the derivative $\dot{N}$.

From "microscopic" point of view there is no fundamental difference between the two mechanisms of $\nu_{e L} \rightarrow \bar{\nu}_{e R}$ conversion discussed above. In the direct case, the intermediate $\nu_{\mu L}\left(\bar{\nu}_{\mu R}\right)$ state is virtual and both the (first-order) flavour and spin-flavour transitions which 
result in the second-order $\nu_{e L}-\bar{\nu}_{e R}$ mixing can in principle occur at the same point. In the two-step conversion the intermediate $\nu_{\mu L}$ state is practically real, the two resonant conversions are separated by a macroscopic distance, and the transition probability can be represented as a product of the individual transition probabilities in the two resonances. If the $\nu_{e L} \leftrightarrow \bar{\nu}_{e R}$ resonance point approaches the $\nu_{e L} \leftrightarrow \nu_{\mu L}$ and $\bar{\nu}_{e R} \leftrightarrow \nu_{\mu L}$ resonances, an

enhancement of the $\nu_{e L}-\bar{\nu}_{e R}$ mixing takes place. In the merging point the direct $\nu_{e L}-\bar{\nu}_{e R}$ mixing is no longer suppressed and the direct $\nu_{e L} \leftrightarrow \bar{\nu}_{e R}$ conversion becomes as efficient as any of the transitions caused by the generic first order mixings present in the effective Hamiltonian in eq. (3). At the same time when $N_{f}$ and $N_{\overline{s f}}$ approach $N_{m}$ the two resonant regions associated with the two transitions in the two-step $\nu_{e L} \rightarrow \bar{\nu}_{e R}$ conversion approach each other and merge; both resonances take place in the same point, thus recovering the case of the direct $\nu_{e L} \rightarrow \bar{\nu}_{e R}$ conversion.

We have studied so far the merging of three resonances when $\dot{\phi}=-4 c_{2} \delta /(1+r)<0$. The case of merging of the $\nu_{\mu L} \leftrightarrow \nu_{e L}, \nu_{e L} \leftrightarrow \bar{\nu}_{\mu R}$, and $\nu_{\mu L} \leftrightarrow \bar{\nu}_{\mu R}$ resonances (Fig. 2f), which can be realized for $\dot{\phi}=4 c_{2} \delta r /(1+r)>0$, can be analyzed along the same lines.

\section{Phenomenological Implications}

We would like to make a few remarks concerning the possible phenomenological implications of the results obtained above. Consider first the consequences for the solar neutrinos.

1. It should be noted that magnetic field configurations in the Sun implying twisting fields were considered in the astrophysical literature without any reference to the solar neutrino problem [16]. What is really to be questioned is whether one should expect the magnitude of the field rotation velocity to be relevant for the evolution of the neutrino system. Let us show that this is really the case. As we have seen, the field rotation effects are important when

$$
\dot{\phi} \sim \sqrt{2} G_{F} N_{e f f}
$$


Consider neutrino spin or spin-flavour precession in the convective zone (precession in the interior regions of the Sun is unable to account for the time variations of the solar neutrino flux). The magnitude of $\dot{\phi}$ can be characterized by the curvature radius $r_{0}$ of the magnetic field lines, $\dot{\phi} \sim 1 / r_{0}$. The depth of the solar convective zone is $\sim 0.3 R_{\odot}$, so that one can expect $r_{0}$ to be of the order of $10 \%$ of the solar radius $R_{\odot}$. This gives $\dot{\phi} \sim 10 / R_{\odot} \simeq 3 \times 10^{-15}$ $\mathrm{eV}$, whereas the r.h.s. of eq. (64) for the density near the bottom of the convective zone $\rho \simeq 0.16 \mathrm{~g} / \mathrm{cc}$ is $\simeq 8 \times 10^{-15} \mathrm{eV}$, i.e. exactly of the same order of magnitude. It is remarkable that such apparently unrelated quantities as the Fermi constant, solar matter density in the convective zone and solar radius turn out to satisfy eq. (64). We would like to emphasize that one does not need any fine tuning of the parameter $\dot{\phi}$ : for each value of $\dot{\phi}$ in the abovedefined range and given neutrino energy $E$ there exists a corresponding value of matter density in the convective zone for which the resonance condition is satisfied.

2. As was pointed out in [12, 13], field rotation effects can either enhance or suppress neutrino spin or spin-flavour precession in the Sun depending on the sign and magnitude of $\dot{\phi}$. It can also change the energy dependence of the $\nu_{e L}$ survival probability and so distort the solar neutrino spectrum. Twisting magnetic field can give rise to some specific effects which could, in principle, be used for identification of the rotation. For example, the picture of the semiannual variations of the solar neutrino flux should crucially depend on the field rotation. These variations can be either enhanced or suppressed, or the minima of the flux can become asymmetric depending on the signs of the $\dot{\phi}$ in the northern and southern solar hemispheres [13, 17]. The field rotation effects can also manifest themselves in additional time dependences of the solar neutrino flux which are not related to the time variation of the strength of the magnetic field (11-yr cycle). This could come about if the field-rotation profile in the Sun $\phi(r)$ is not constant in time. Another possible reason for short-time variations can be related to the rotation of the Sun and azimuthal dependence of the $\dot{\phi}$ profile. In this case one can expect the neutrino flux to vary in time with a period comparable to the solar day ( $\simeq 27$ earth days). 
3. The permutation of the resonances will lead to a change of the helicity and the flavour content of the neutrino final state. If the adiabaticity conditions hold, the change of the order of the MSW and the spin-flavour resonances will lead to the presence of a considerable $\nu_{\mu L}$ component in the neutrino flux at Earth, instead of a $\bar{\nu}_{\mu R}$ component. Unfortunately, this two possible components of the neutrino flux cannot be distinguished in the current and next generation experiments with solar (and supernova) neutrinos: the neutral current interactions of the low energy $(E \lesssim 50 \mathrm{MeV}) \nu_{\mu L}$ and $\bar{\nu}_{\mu R}$ are practically the same.

4. The most interesting results of our study are associated with the $\nu_{e L} \rightarrow \bar{\nu}_{e R}$ transition. As we have shown, magnetic field rotation can modify drastically this transition: it can strongly enhance or suppress the $\nu_{e L} \rightarrow \bar{\nu}_{e R}$ conversion probability and, consequently, the $\bar{\nu}_{e R}$ component of the neutrino flux from the Sun. In particular, the field rotation can induce direct $\nu_{e L} \rightarrow \bar{\nu}_{e R}$ resonant conversion, make the two-step conversion resonant, and lead to the phenomenon of merging of resonances. In the latter cases a strong $\nu_{e L} \rightarrow \bar{\nu}_{e R}$ conversion is possible. The conditions for direct isolated $\nu_{e L} \rightarrow \bar{\nu}_{e R}$ transitions are more restrictive than those for the usual RSFP. In particular, given the same magnetic field and matter density distributions, the adiabaticity parameter for the former is generally smaller. Thus, even if the RSFP conversion is efficient in the Sun, the direct $\nu_{e L} \rightarrow \bar{\nu}_{e R}$ transition may not be efficient. However, the adiabaticity condition in the triple resonance can be fulfilled in the Sun even if the transition in the isolated $\nu_{e L} \rightarrow \bar{\nu}_{e R}$ resonance is non-adiabatic. This condition and the relevant adiabaticity parameters at the merging point depend on the neutrino energy. Thus, one can expect an enhancement of the $\bar{\nu}_{e R}$ component of the solar neutrino flux in a definite energy range. A sizable flux of $\bar{\nu}_{e R}$ from the Sun would be in contradiction with the upper limit $\Phi\left(\bar{\nu}_{e}\right) \lesssim(5-7) \% \Phi\left(\nu_{e}\right)$ obtained from the analyses of the Kamiokande II and LSD data [18, 19], and so some regions of values of the relevant parameters can be ruled out. Note, however, that these constraints are valid only if the $\nu_{e L}$ are produced at a density $N_{0}<N_{s f}$ or if the $\nu_{e L} \rightarrow \bar{\nu}_{\mu R}$ RSFP conversion is non-adiabatic. This requires either small neutrino energies or large values of $\Delta m^{2}$; in the first case the limit on the flux of solar $\bar{\nu}_{e}$ obtained from the data of the Kamiokande and LSD experiments, 
which are only sensitive to the high energy ${ }^{8} \mathrm{~B}$ neutrinos, may not be applicable. This means that the upper bound on the flux of solar $\bar{\nu}_{e}$ may be used to exclude some values of $\dot{\phi}$ only for large enough values of $\Delta m^{2}, \Delta m^{2} \gtrsim 2 \times 10^{-7} \mathrm{eV}^{2}$.

The resonant $\nu_{e} \rightarrow \bar{\nu}_{e}$ conversion can be compatible with the existing upper bound on the $\bar{\nu}_{e}$ flux from the Sun since the transition may not be adiabatic. It is therefore important to look for the $\bar{\nu}_{e}$ flux in such solar neutrino experiments as SNO, Borexino and Super-Kamiokande which are expected to be very sensitive to $\bar{\nu}_{e}$ 's.

One should stress that $\bar{\nu}_{e}$ 's can be produced by the Sun even in the non-resonant case when $\dot{\phi}=0$ [6, 15]; however the resonant $\nu_{e} \rightarrow \bar{\nu}_{e}$ conversion increases the $\bar{\nu}_{e}$ flux significantly and also has a clear signature which allows one to distinguish this mechanism from the others: the flux of $\bar{\nu}_{e}$ 's should have a peak at the energy defined by the condition $\sin 2 \omega \cong 1$ $\left(s_{2} \delta \cong \mu B_{\perp}\right)$, whereas the energy spectra of $\bar{\nu}_{e}$ 's generated by other mechanisms (including non-resonant RSFP+neutrino oscillations) should be smooth. It is important to notice that the position and the width of the peak strongly depend on the magnetic field strength in the region of the resonance; therefore the energy spectrum of resonantly emitted solar $\bar{\nu}_{e}$ 's should exhibit characteristic time dependence (in particular, the energy at which the $\bar{\nu}_{e}$ spectrum achieves its maximum should vary in time).

Obtaining experimental information on the relative fluxes and the energy spectra (and their time dependences) of the $\nu_{e}, \bar{\nu}_{e}$ and $\left(\nu_{\mu}+\bar{\nu}_{\mu}+\nu_{\tau}+\bar{\nu}_{\tau}\right)$ components of the solar neutrino flux will be crucial for testing the above results [20].

Magnetic field rotation can also be important for the transitions of the supernova neutrinos. The field rotation in the supernovae can be induced by differential rotation of matter. As we have seen, it influences the order of the resonances which in turn will determine the fate of various neutrino species propagating in a collapsing star. Since neutrinos of different species have in general different mean energies, their transmutations can have observable consequences [4, 21]. This issue will be considered in detail elsewhere. 


\section{Conclusions}

For a system of flavour neutrinos with vacuum mixing and flavour-off-diagonal magnetic moments, the chirality dependent level shifts induced by the magnetic field rotation give rise to qualitatively new effects. The energy level scheme can be changed drastically by the magnetic field rotation. The location of and the distances between the resonances are changed. Two new types of resonances appear at nonzero densities, namely, resonances in the neutrino-antineutrino transitions $\nu_{e L} \leftrightarrow \bar{\nu}_{e R}$ and $\nu_{\mu L} \leftrightarrow \bar{\nu}_{\mu R}$. For a definite value of the magnetic field rotation velocity $\dot{\phi}$ (or of the neutrino energy) merging of three resonances in one point can take place. The types of resonances that merge depend on the sign of $\dot{\phi}$ : for $\dot{\phi}<0$, the $\nu_{e L} \leftrightarrow \nu_{\mu L}, \bar{\nu}_{e R} \leftrightarrow \nu_{\mu L}$ and $\nu_{e L} \leftrightarrow \bar{\nu}_{e R}$ resonances can occur at one point, whereas for $\dot{\phi}>0$, the $\nu_{e L} \leftrightarrow \nu_{\mu L}, \nu_{e L} \leftrightarrow \bar{\nu}_{\mu R}$ and $\nu_{\mu L} \leftrightarrow \bar{\nu}_{\mu R}$ resonances can merge (assuming $\left.c_{2} \delta>0\right)$. For sufficiently large values of $|\dot{\phi}|$ permutation of the resonances occurs.

The resonant $\nu_{e L} \rightarrow \bar{\nu}_{e R}$ transitions can proceed in two ways: i) direct $\nu_{e L} \rightarrow \bar{\nu}_{e R}$ resonant conversion induced by second order $\nu_{e L}-\bar{\nu}_{e R}$ mixing; ii) two-step $\nu_{e L} \rightarrow \bar{\nu}_{e R}$ conversion which proceeds via two consecutive resonant conversions $\nu_{e L} \rightarrow \nu_{\mu L}$ and $\nu_{\mu L} \rightarrow$ $\bar{\nu}_{e R}$ taking place in different regions separated by a macroscopic distance. Either of these mechanisms can dominate in the $\nu_{e L} \rightarrow \bar{\nu}_{e R}$ transitions, depending on the values of the parameters $c_{2} \delta, \dot{\phi}, \mu B_{\perp}$ and $N_{0}$.

In the case of merging of three resonances the indicated two mechanisms of $\nu_{e L} \rightarrow \bar{\nu}_{e R}$ conversion also "merge" becoming indistinguishable. A complete $\nu_{e L} \rightarrow \bar{\nu}_{e R}$ conversion in one resonance region is possible in this case.

In a medium with constant $s_{2} \delta, \mu B_{\perp}$ and $\dot{\phi}$ having the values for which the three resonances merge, or the isolated $\nu_{e L} \rightarrow \bar{\nu}_{e R}$ resonance occurs, the oscillation-precession of $\nu_{e L}$ into its $C P$-conjugate state $\bar{\nu}_{e R}$ can proceed with maximal depth.

The effects discussed above can exist for solar neutrinos and for the neutrinos emitted by collapsing stars. 


\section{Acknowledgements}

The authors are grateful to M. Moretti for discussions at the early stage of the present study. A.Yu.S. would like to thank Prof. A. Salam, the International Atomic Energy Agency and UNESCO for hospitality at the International Centre for Theoretical Physics. The work of S.T.P. was supported in part by the Bulgarian National Science Foundation via grant PH-16. 


\section{References}

[1] A. Cisneros, Astrophys. Space Sci. 10 (1970) 87.

[2] K. Fujikawa, R.E. Shrock, Phys. Rev. Lett. 45 (1980) 963.

[3] M.B. Voloshin, M.I. Vysotsky, Sov. J. Nucl. Phys. 44 (1986) 845; M.B. Voloshin, M.I. Vysotsky, L.B. Okun, Sov. Phys. JETP 64 (1986) 446.

[4] J. Schechter, J.W.F. Valle, Phys. Rev. D24 (1981) 1883; Phys. Rev. D25 (1982) 283 (E).

[5] E.Kh. Akhmedov, Sov. J. Nucl. Phys. 48 (1988) 382; Phys. Lett. B213 (1988) 64.

[6] C.-S. Lim, W.J. Marciano, Phys. Rev. D37 (1988) 1368.

[7] S.P. Mikheyev, A.Yu. Smirnov, Sov. J. Nucl. Phys. 42 (1985) 913; Prog. Part. Nuc. Phys. 23 (1989) 41.

[8] L. Wolfenstein, Phys. Rev. D17 (1978) 2369.

[9] E.Kh. Akhmedov, Phys. Lett. B257 (1991) 163.

[10] J. Vidal, J. Wudka, Phys. Lett. B249 (1990) 473.

[11] C. Aneziris, J. Schechter, Int. J. Mod. Phys. A6 (1991) 2375.

[12] A.Yu. Smirnov, Phys. Lett. B260 (1991) 161.

[13] E.Kh. Akhmedov, P.I. Krastev, A.Yu. Smirnov, Z. Phys. C52 (1991) 701.

[14] C. Aneziris, J. Schechter, Phys. Rev. D45 (1992) 1053.

[15] E.Kh. Akhmedov, Sov. Phys. JETP 68 (1989) 690.

[16] Y. Nakagawa, In Solar Magnetic Fields, ed. by R. Howard, Springer-Verlag, New York, 1971. 
[17] T. Kubota, T. Kurimoto, M. Ogura, E. Takasugi, Phys. Lett. B 292 (1992) 195.

[18] R. Barbieri, G. Fiorentini, G. Mezzorani, M. Moretti, Phys. Lett. B259 (1991) 119.

[19] LSD Collaboration, M. Aglietta et al., preprint ICGF 269/92.

[20] E.Kh. Akhmedov, S.T. Petcov, A.Yu. Smirnov, in preparation.

[21] E.Kh. Akhmedov, Z.G. Berezhiani, Nucl. Phys. B373 (1992) 479. 


\section{Figure Captions}

Figure 1. Neutrino energy levels versus density $N$ for $\dot{\phi}=0$ and $c_{2} \delta>0$. The $\nu_{e L}$ and $\nu_{\mu L}$ levels are shown as solid lines, the $\bar{\nu}_{\mu R}$ and the $\bar{\nu}_{e R}$ levels as dash-dotted and dashed lines, respectively. Dotted lines represent the energy levels of the eigenstates of the Hamiltonian.

Figure 2. Neutrino energy levels versus $N$ for different values of the magnetic field rotation velocity $\dot{\phi}$. The notations are the same as in Fig. 1. Figures $a-d$ correspond to the case of $\dot{\phi}<0$, and figures $e-h$ correspond to $\dot{\phi}>0$; the consecutive figures $a-d$ and $e-h$ have been obtained by increasing the value of $|\dot{\phi}|$ (see the text for details). The $\nu_{e L} \rightarrow \bar{\nu}_{\mu R}$ resonance is not shown in fugures $a-d$ because of lack of space.

Figure 3. The level-crossing scheme for isotopically neutral medium $(\mathrm{r}=1)$. The notations are the same as in Fig. 1.

Figure 4. The dependence of the neutrino energy levels on density in the case of triple resonance merging $\left(\dot{\phi}=-4 c_{2} \delta /(1+r)<0\right)$ for: a) critical value of the ratio $s_{2} \delta /\left(\mu B_{\perp}\right)$ (see eq. (51)), b) weak flavour mixing, $\tan \omega<\tan \omega_{c}$, and c) strong flavour mixing, $\tan \omega>\tan \omega_{c}$. The notations are the same as in Fig. 1. 